\title{
Oral bisphosphonates improve the bone mineral density in men with diabetes with or without thiazolidinediones
}

\author{
Subhashini Yaturu $^{1^{*}}$, Jared Davis $^{2}$ \\ ${ }^{1}$ Section of Endocrinology and Metabolism, Stratton VA Medical Center, Albany, USA; \\ *Corresponding Author: Subhashini.Yaturu@,va.gov; yaturu@yahoo.com \\ ${ }^{2}$ Overton Brooks VA Medical Center, Shreveport, USA.
}

Received 23 August 2011; revised 29 September 2011; accepted 9 October 2011.

\begin{abstract}
Background: Osteoporosis and type 2 diabetes mellitus (DM) are two of the most common chronic conditions and represent major public health burdens. Epidemiological and observational studies indicate that thiazolidinedione (TZD) therapy with rosiglitazone and pioglitazone is associated with an increased risk of fractures and decreased bone mineral density (BMD). To our knowledge, no data are available to evaluate bisphosphonate therapy in TZD treated patients. Aim: The aim of this study was to investigate the benefit of bisphosphonates to improve changes in BMD associated with the use of TZDs in subjects with DM. Methods: In a cross-sectional, observational study using a retrospective review of electronic mfedical records, the changes in BMD were compared in subjects with type 2 DM. The study subjects were divided into four groups. First group is with DM receiving both TZDs and BPs; second group TZD use only; third group receiving neither TZDs nor BPs and the fourth only BPs. The study compared the annual percent changes in BMD between the groups. Results: Decreased BMD was noted in subjects with DM on TZDs. Bisphosphonate use improved BMD in subjects with DM on TZDs. BMD improved in subjects with DM in those not receiving TZDs also. Conclusion: We conclude that concomitant treatment with bisphosphonates improves BMD in subjects with diabetes and on TZDs.
\end{abstract}

Keywords: Diabetes; Thiazolidinediones; Bone Mineral Density; Bisphosphonates; Pioglitazone; Rosiglitazone; Osteoporosis

\section{INTRODUCTION}

Subjects with diabetes (DM) have a higher risk of hip fractures [1-6] as well as fractures at other sites [5,7-8]. Fractures are a major cause of morbidity and mortality, [9] resulting in substantial health care and social costs [10-11]. The mortality rate in the first year after a patient sustains a fracture of the neck of the femur is about $36 \%$ in men and $21 \%$ in women [12]. Thiazolidinediones (TZDs) are important therapeutic agents for use in treating type 2 diabetes (DM), with a plethora of beneficial effects in addition to improving hyperglycemia. However, epidemiological and observational studies indicate that TZD therapy with rosiglitazone and pioglitazone is associated with an increased risk of fractures [13-18]. Retrospective and short-term prospective studies have shown that both pioglitazone [19] and rosiglitazone [20] reduce bone mineral density an effect similar to that observed during treatment with glucocorticoids, in which decreased bone formation occurs as a result of inappropriately stable bone resorption [21]. Bone loss is a potent predictor of fracture risk, indicating that TZD use may be associated with a measurable burden on skeletal health. Prevention of osteoporosis requires not only recognition of populations at risk, but also preventive programs targeting them. The aim of our study is to evaluate the benefit of bisphosphonate therapy in TZD-treated patients.

\section{METHODS}

In a retrospective study approved by the Institutional Review Board for the ethical treatment of human research subjects, we reviewed the medical records and BMD studies of subjects with type 2 diabetes who had at least two measurements. The study included 250 subjects with type 2 diabetes. The clinical information collected included age, body mass index (BMI), smoking status, alcohol intake, associated medical conditions and medications that alter bone metabolism, duration of diabetes, history of hypertension, whether being treated with a thiazolidinedione (either pioglitazone or rosiglitazone), and use of bisphosphonates (residronate, alen- 
dronate or zoledronic acid).

\subsection{Biochemical Parameters}

Data on fasting lipid panel, fasting plasma glucose (FPG), hemoglobin A1C (HbA1C), blood urea nitrogen (BUN), serum creatinine $(\mathrm{Cr})$, serum alkaline phosphatase (ALP), and serum calcium (Ca) were also collected.

The ages of the subjects ranged from 50 to 80 years, with a mean age of 65 years. Exclusion criteria included subjects with a potential for increased bone loss such as those with chronic kidney disease, inflammatory disorders like rheumatoid arthritis, or systemic lupus erythematosis (SLE) with abnormal cytokine values. Subjects with a history of malabsorption, chronic pancreatictis or pancreatectomy, chronic steroid use, primary hyperparathyroidism, untreated subclinical or clinical hyperthyroidism and Paget's disease, and those receiving corticosteroids, gonadotropin releasing hormone $(\mathrm{GnRH})$ agonists, gonadal hormones, immunosuppressant medications or anticonvulsants were excluded. Since bone metabolism alters during peri-menopause and menopause (the age range of the study population), female subjects were also excluded from the study.

\subsection{BMD Measurements}

$\operatorname{BMD}\left(\mathrm{g} / \mathrm{cm}^{2}\right)$ was measured at the lumbar spine and femoral neck by DXA using a LUNAR DPX densitometer (GE-LUNAR, Madison, WI, USA). The radiation dose with this method is $<0.1 \mu \mathrm{Gy}$. Based on the actual measurement of femoral neck BMD (FNBMD), data were classified as "osteoporosis" (BMD 2.5 SD or more below the young normal level), "osteopenia" (BMD between $<2.5$ and 1.0 SD below the young normal level), or "normal BMD" (values at $<1.0 \mathrm{SD}$ below the young normal level).

\subsection{Statistical Analysis}

All parameters are expressed as mean \pm SD for each group. The differences between the two experimental groups were determined by performing a one-way analysis of variance (ANOVA). Student's t-test was used for comparison of the data. A $p$-value of $<0.05$ is considered significant.

\section{RESULTS}

The data from 250 subjects with type 2 diabetes are divided into 4 groups based on their TZD and/or bisphosphonate use. Group 1: those receiving both TZD and bisosphonates; Group 2: those receiving TZDs only; Group 3: those receiving neither TZD nor bisphosphotes; and Group 4: those receiving bisphosphonates only. The baseline parameters for each of the four groups are shown in Table 1.

The follow up period varied from 1.4 to 2.8 years. Since the follow up period is not uniform, we calculated the percentage change in BMD to correct for the varied follow up periods, and converted the data to a uniform distribution. The change in BMD in all the four groups is shown in Table 2. An improvement in BMD was noted in both groups on bisphosphonates regardless of TZD use. The TZD group without bisphosphonates had a deeased BMD of $1.09 \%$ at the femoral neck, $0.87 \%$ at total hip and $1.09 \%$ at 0.33 radius.

Table 1. Baseline characteristics.

\begin{tabular}{ccccc}
\hline & $\begin{array}{c}\text { TZD + BPs } \\
(25)\end{array}$ & TZD (38) & $\begin{array}{c}\text { Neither } \\
(124)\end{array}$ & $\begin{array}{c}\text { BPs + No } \\
\text { TZD (63) }\end{array}$ \\
\hline Age (yr) & 69 & 63 & 63 & 69 \\
Years F/U & 2.56 & 1.47 & 1.91 & 2.24 \\
BMI (kg/m $\left.{ }^{2}\right)$ & 31.14 & 32.36 & 31.16 & 30.26 \\
HbA1C & 7.3 & 7.8 & 7.0 & 6.99 \\
25 OHD (ng/ml) & 28 & 22 & 27 & 23 \\
1.25 Vitamin D (pg/ml) & 36.9 & 33 & 29 & 31 \\
\hline
\end{tabular}

TZD: thiazolidinedione; BP: bisphosphonates; BMI: body mass index; F/U: follow up in years.

Table 2. Changes in Bone mineral density at follow up.

\begin{tabular}{|c|c|c|c|c|c|c|c|c|}
\hline & \multicolumn{2}{|c|}{ TZD + BPs ( 25) } & \multicolumn{2}{|c|}{ TZD (38) } & \multicolumn{2}{|c|}{ Neither (124) } & \multicolumn{2}{|c|}{ BPs + No TZD (63) } \\
\hline & Baseline BMD & $\begin{array}{l}\text { Annual } \\
\% \text { change }\end{array}$ & Baseline BMD & $\begin{array}{l}\text { Annual } \\
\% \text { change }\end{array}$ & Baseline BMD & $\begin{array}{l}\text { Annual } \\
\% \text { change }\end{array}$ & Baseline BMD & $\begin{array}{l}\text { Annual } \\
\% \text { change }\end{array}$ \\
\hline AP Spine $\left(\mathrm{g} / \mathrm{cm}^{2}\right)$ & 1.140 & 1.15 & 1.24 & 0.69 & 1.22 & 1.1 & 1.161 & 2.2 \\
\hline $\operatorname{Neck}\left(\mathrm{g} / \mathrm{cm}^{2}\right)$ & 0.849 & $1.31 *$ & 0.964 & -1.09 & 0.927 & -0.01 & 0.852 & 2.02 \\
\hline Total Hip $\left(\mathrm{g} / \mathrm{cm}^{2}\right)$ & 0.917 & 2.06 & 1.050 & -0.87 & 1.006 & -0.08 & 0.902 & 2.02 \\
\hline Radius:0.33 $\left(\mathrm{g} / \mathrm{cm}^{2}\right)$ & 0.737 & $2.54^{*}$ & 0.875 & $-1.09 *$ & 0.868 & $-1.17 *$ & 0.759 & $1.93 *$ \\
\hline
\end{tabular}

BMI: body mass index; TZD: thiazolidinedione; BP: bisphosphonates; BMD: bone mineral density; *Significant. 


\section{DISCUSSION}

The literature is limited on the effect of anti resorptive agents in subjects with diabetes in general. This is the first study to evaluate the effect of anti resorptive agents on BMD in subjects with diabetes being treated with TZDs. The main findings of this study were: 1) Improvement in BMD with the use of BPs in subjects with diabetes on TZDs; 2) the response to BPs in diabetic subjects taking TZDs is similar to those taking BPs but not TZDs at spine and total hip; 3 ) the response to BPs in diabetic subjects taking TZDs is lower compared to that of those taking BPs but not TZDs at femoral neck. In a retrospective Danish study conducted by Vestergaard and associates, no difference was observed in the antifracture efficacy of treatment with bisphosphonates and raloxifene between patients with diabetes and nondiabetic controls, or between patients with type 1 or type 2 diabetes [22]. Dagdelen and associates evaluated BMD response to alendronate in women with concurrent late postmenopausal osteoporosis and type 2 diabetes in a retrospective study [23]. They noted no difference in spinal BMD response to alendronate therapy in the presence of type 2 DM. In contrast, BMD in the total hip (mean percentage change in $\mathrm{BMD},-5.6 \% \mathrm{vs}+1.4 \% ; P=$ $0.096)$, femoral neck $(-8.1 \%$ vs $+1.1 \% ; P=0.015)$, and forearm $(-3.6 \%$ vs $+12.7 \%$; $P=0.013)$ fell progressively from baseline in subjects with type $2 \mathrm{DM}$ who were taking alendronate for 4.8 years, compared with controls [23]. Based on their results, they concluded that elderly, postmenopausal, osteoporotic obese women with type $2 \mathrm{DM}$ are resistant to long-term bisphosphonates, especially in the hip, femoral neck, and forearm as compared with the spine.

Bisphosphonates, the most commonly used treatment for established osteoporosis, inhibit osteoclast-mediated bone resorption and reduce the risk of vertebral fracture.

Though oral bisphosphonates have been shown to reduce non-vertebral and hip fractures, adherence to oral treatment is problematic, and about half of patients for whom oral treatment is prescribed do not adhere to it after 1 year $[24,25]$, which compromises the effectiveness of treatment [26,27]. Their low bioavailability and low potency necessitate frequent administration on an empty stomach, which may reduce compliance. Compliance could not be addressed as the study was retrospecttive. In a retrospective study to evaluate whether diabetes mellitus (DM) may contribute to development of osteonecrosis of the jaw, it was suggested that patients with diabetes treated with bisphosphonates should be carefully monitored [28]. It is important to note that these subjects were receiving parenteral bisphosphonates for malignancy related causes and more frequently. In a randomized control pilot study of the effect of oral alen- dronate once a week on acute Charcot neuropathy, the authors noted that there was a significant reduction of ICTP and hydroxyprolin, markers indicative of bone resorption [29]. Although the purpose of the study was to evaluate the benefit of bisphosphonate treatment on $\mathrm{Ch}$ arcot's disease, it also demonstrated an improvement in the bone turnover markers in subjects with diabetes on oral bisphosphonates. In a small Japanese study that examined changes in biochemical markers of bone turnover and BMD in a response to alendronate versus alfacalcidol treatment in postmenopausal women with type 2 diabetes, urinary $\mathrm{N}$-telopeptide was found to have decreased markedly with no significant change in BMD with the use of alendronate [30].

\subsection{Limitations of the Study}

Compare the values for bone turnover markers as they were not available for most of the subjects. The data groups are not large enough to perform regression analyses for confounding factors such as age, duration of diabetes, use of insulin, association of hypertension, or other factors such as smoking or alcoholism. Other confounding factors such as hypogonadism and drug induced bone changes could not be evaluated and the regression analysis could not be carried out for these potential confounders. Compliance with the medication (bisphosphonate) could not be evaluated. Baseline BMD data are not comparable in the four groups as the percentage changes depend on the baseline BMD. Some of the subjects had osteopenia rather than osteoporosis. Nevertheless, this study shows a significant increase in bone loss in type 2 diabetic men on rosiglitazone treatment and improvement on BPs.

\subsection{Conclusion}

Bisphosphonates improve TZD induced bone loss.

\section{ACKNOWLEDGEMENTS}

Both Dr. Yaturu and Mr. Davis receive salary support from VAMC. Dr. Yaturu had a VA Merit Review Grant at the time of the study.

\section{REFERENCES}

[1] Strotmeyer, E.S. and Cauley, J.A. (2007) Diabetes mellitus, bone mineral density, and fracture risk. Current Opinion in Endocrinology, Diabetes and Obesity, 14, 429-435. doi:10.1097/MED.0b013e3282f1cba3

[2] Janghorbani, M., et al. (2006) Prospective study of diabetes and risk of hip fracture: The nurses' health study. Diabetes Care, 29, 1573-1578. doi:10.2337/dc06-0440

[3] Strotmeyer, E.S., et al. (2005) Nontraumatic fracture risk with diabetes mellitus and impaired fasting glucose in older white and black adults: The health, aging, and body 
composition study. Archives of Internal Medicine, 165 , 1612-1617. doi:10.1001/archinte.165.14.1612

[4] Cauley, J.A., et al. (2005) Bone mineral density and the risk of incident nonspinal fractures in black and white women. The Journal of the American Medical Association, 293, 2102-2108. doi:10.1001/jama.293.17.2102

[5] Schwartz, A.V. and Sellmeyer, D.E. (2004) Women, type 2 diabetes, and fracture risk. Current Diabetes Reports, 4, 364-369. doi:10.1007/s11892-004-0039-z

[6] Nicodemus, K.K. and Folsom, A.R. (2001) Type 1 and type 2 diabetes and incident hip fractures in postmenopausal women. Diabetes Care, 24, 1192-1197. doi:10.2337/diacare.24.7.1192

[7] Wallace, C., et al. (2002) Incidence of falls, risk factors for falls, and fall-related fractures in individuals with diabetes and a prior foot ulcer. Diabetes Care, 25, 1983-1986. doi:10.2337/diacare.25.11.1983

[8] Schwartz, A.V., et al. (2002) Older women with diabetes have a higher risk of falls: A prospective study. Diabetes Care, 25, 1749-1754. doi:10.2337/diacare.25.10.1749

[9] Keene, G.S., Parker, M.J. and Pryor, G.A. (1993) Mortality and morbidity after hip fractures. British Medical Journal, 307, 1248-1250. doi:10.1136/bmj.307.6914.1248

[10] Haentjens, P., et al. (2001) The economic cost of hip fractures among elderly women. A one-year, prospective, observational cohort study with matched-pair analysis. Belgian Hip Fracture Study Group. The Journal of Bone and Joint Surgery (American Volume), 83-A, 493-500.

[11] Hollingworth, W., et al. (1993) Cost analysis of early discharge after hip fracture. British Medical Journal, 307, 903-906. doi:10.1136/bmj.307.6909.903

[12] Botushanov, N.P. and Orbetzova, M.M. (2009) Bone mineral density and fracture risk in patients with type 1 and type 2 diabetes mellitus. Folia Medica (Plovdiv), 51, 12-17.

[13] Kahn, S.E., et al. (2006) Glycemic durability of rosiglitazone, metformin, or glyburide monotherapy. The New England Journal of Medicine, 355, 2427-2443. doi:10.1056/NEJMoa066224

[14] Douglas, I.J., et al. (2009) The risk of fractures associated with thiazolidinediones: A self-controlled case-series study. PLoS Medicine, 6, e1000154. doi:10.1371/journal.pmed.1000154

[15] Jones, S.G., et al. (2009) Distal upper and lower limb fractures associated with thiazolidinedione use. American Journal of Managed Care, 15, 491-496.

[16] Dormuth, C.R., et al. (2009) Thiazolidinediones and fractures in men and women. Archives of Internal Medicine, 169, 1395-1402.

doi:10.1001/archinternmed.2009.214

[17] Bodmer, M., et al. (2009) Risk of fractures with glitazones: A critical review of the evidence to date. Drug Safety, 32, 539-547. doi:10.2165/00002018-200932070-00001

[18] Bilik, D., et al. (2010) Thiazolidinediones and fractures:
Evidence from translating research into action for diabetes. The Journal of Clinical Endocrinology \& Metabolism, 95, 4560-4565.

[19] Glintborg, D., et al. (2008) Association of pioglitazone treatment with decreased bone mineral density in obese premenopausal patients with polycystic ovary syndrome: A randomized, placebo-controlled trial. The Journal of Clinical Endocrinology \& Metabolism, 93, 1696-1701. doi:10.1210/jc.2007-2249

[20] Yaturu, S., Bryant, B. and Jain, S.K. (2007) Thiazolidinedione treatment decreases bone mineral density in type 2 diabetic men. Diabetes Care, 30, 1574-1576. doi: $10.2337 / \mathrm{dc} 06-2606$

[21] Ton, F.N., et al. (2005) Effects of low-dose prednisone on bone metabolism. Journal of Bone and Mineral Research, 20, 464-470. doi:10.1359/JBMR.041125

[22] Vestergaard, P., Rejnmark, L. and Mosekilde, L. (2010) Are antiresorptive drugs effective against fractures in patients with diabetes? Calcified Tissue International, 88, 209-214.

[23] Dagdelen, S., Sener, D. and Bayraktar, M. (2007) Influence of type 2 diabetes mellitus on bone mineral density response to bisphosphonates in late postmenopausal osteoporosis. Advances in Therapy, 24, 1314-1320. doi:10.1007/BF02877778

[24] Lo, J.C., et al. (2006) Persistence with weekly alendronate therapy among postmenopausal women. Osteoporosis International, 17, 922-928. doi:10.1007/s00198-006-0085-2

[25] Cramer, J.A., et al. (2005) Compliance and persistence with bisphosphonate dosing regimens among women with postmenopausal osteoporosis. Current Medical Research \& Opinion, 21, 1453-1460. doi:10.1185/030079905X61875

[26] Siris, E.S., et al. (2006) Adherence to bisphosphonate therapy and fracture rates in osteoporotic women: Relationship to vertebral and nonvertebral fractures from 2 US claims databases. Mayo Clinic Proceedings, 81, 1013-1022. doi:10.4065/81.8.1013

[27] Huybrechts, K.F., Ishak, K.J. and Caro, J.J. (2006) Assessment of compliance with osteoporosis treatment and its consequences in a managed care population. Bone, 38, 922-928. doi:10.1016/j.bone.2005.10.022

[28] Khamaisi, M., et al. (2007) Possible association between diabetes and bisphosphonate-related jaw osteonecrosis. The Journal of Clinical Endocrinology \& Metabolism, 92, 1172-1175. doi:10.1210/jc.2006-2036

[29] Pitocco, D., et al. (2005) Six-month treatment with alendronate in acute Charcot neuroarthropathy: A randomized controlled trial. Diabetes Care, 28, 1214-1245. doi:10.2337/diacare.28.5.1214

[30] Ikeda, T., Manabe, H. and Iwata, K. (2004) Clinical significance of alendronate in postmenopausal type 2 diabetes mellitus. Diabetes \& Metabolism, 30, 355-358. doi:10.1016/S1262-3636(07)70128-3 